\title{
perifèria
}

Número 19 (2), Diciembre 2014

revistes.uab.cat/periferia

\section{Reseña de "El Capital en el Siglo XXI", de Thomas}

\section{Piketty}

\author{
Marta Lobato - egolab-GRAFO, Departamento de Antropología Social y \\ Cultural, Universitat Autònoma de Barcelona ${ }^{1}$
}

DOI: http://dx.doi.org/10.5565/rev/periferia.443

\section{Resumen}

El siguiente artículo es una reseña crítica sobre el libro del economista Thomas Piketty, "Le capital au XXIème siècle" (2013), el cual ha sido recibido por diversas disciplinas dentro de la comunidad académica como una obra de gran importancia dentro del actual marco político, económico y social. Piketty trata el tema de la creciente desigualdad de riqueza y por tanto, de la diferencia de clases, combinando nociones de la economía clásica con un mensaje contundente: el capitalismo por sí sólo tiende de manera natural a crear desigualdad. Con un título que hace una clara referencia al "Das Kapital" de Karl Marx, se reabren los debates clásicos de las ciencias sociales.

\begin{abstract}
The following article is a critical review of the worldwide acclaimed book "Capital in the $21^{\text {st }}$ Century" (2013), which has been received by different disciplines within the academic world as a book of great importance within the current political, economic and social scenery. Piketty speaks of the rising inequality around the world, and about the increasing gap amongst classes by combining classical notions of economics with a strong message; capitalism left on its own tends to create inequality. By using a title that reassembles Karl Marx's "Das Kapital", classical debates within the social sciences have reopened.
\end{abstract}

\section{Introducción}

El éxito de la obra de Thomas Piketty, El Capital en el siglo XXI solamente tiene un parangón en el mismísimo Das Kapital de Carlos Marx. El volumen de publicaciones (digitales o no) que se han sucedido tras su aparición en francés es especialmente sorprendente si se tiene en cuenta que se refieren a una publicación académica de

\footnotetext{
${ }^{1}$ Marta M. Lobato Rodríguez (martamaria.lobato@uab.cat)
} 


\section{perifèria}

Número 19 (2), Diciembre 2014

revistes.uab.cat/periferia

más de 600 páginas. Este acalorado debate es importante por al menos dos razones. La primera, porque es un indicativo de la existencia de un clamoroso vacío en los análisis económicos, la desigualdad de las "clases sociales", tema relegado durante un largo período de crecimiento económico y de ideología neoliberal. En segundo lugar, porque no deja de ser desconcertante que un economista, de l'École Des Hautes Études en Sciences Sociales (EHESS) y profesor del Paris School of Economics, hable de desigualdad, riqueza e impuestos a las grandes fortunas.

La aparición de este libro en España coincide además con un momento de profunda crisis económica ligada a numerosos casos de corrupción política que han hecho especialmente oportuna su explicación de la realidad existente: el crecimiento imparable de las rentas del capital en detrimento de las rentas del trabajo.

La revista Real-World Economics Review ha publicado recientemente un volumen especial dedicado precisamente a glosar la obra de Piketty. En este volumen no faltan críticas feroces hacia el "desmesurado" éxito cosechado. Wade y Parker (2014) por ejemplo, lo tachan de "convencional" y "poco revelador" (p.8 y p.59) en cuanto a las ideas básicas sobre las cuales se sustenta su teoría. Yanis Varoufakis critica las tres leyes fundamentales sobre las que Piketty apoya sus fórmulas matemáticas; de ellas argumenta que la primera es una tautología, la segunda una "suposición heroica" y la tercera una mera "trivialidad" (p. 19).

Sin embargo, y admitiendo que hay espacio para el debate, el hecho es que la desigualdad ha crecido de forma imparable desde finales de la década de los 70, como diversos autores ajenos a Piketty ${ }^{2}$ han mencionado. ¿Qué implicaciones tiene la creciente desigualdad de clases en términos generacionales, relaciones entre centro y periferia, norte-sur, o en términos de cohesión social y estratificación social? O como explica Wade, el libro se concentra mucho en rentas y distribución

2 James Galbraith, por ejemplo, escribió en 2012 "Inequality and Instability: A Study of the World Economy Just Before the Great Crisis" y tiene otras publicaciones que giran en torno al tema de la desigualdad. Robert Kuttner, the Economic Policy Institute, Edward Wolff, et al. Gabriel Kolko, Wealth and Power in America. Wade, R.H. "Global Trends in Income Inequality" (2011) y "Why has income inequality remained on the sidelines of public policy for so long?" (2012) 


\section{perifèria}

Número 19 (2), Diciembre 2014

revistes.uab.cat/periferia

de riqueza, pero dice poco sobre los procesos productivos, por ejemplo sobre las estructuras de poder dentro del mundo del trabajo, y especialmente sobre las relaciones entre propietarios del capital y el resto (p. 8). Asimismo, ¿qué podemos decir de la economía doméstica y moral de las grandes élites, o de esta "nueva" clase media acaudalada, protagonista del "capitalismo de patrimonio" del que habla Piketty? ¿Qué transferencias de poder, de valores, y de dinero se están dando dentro de este segmento de la población?

\section{La perspectiva y metodología de Piketty}

Piketty comienza su libro "El Capital en el siglo XXI" exponiendo la idea principal que se propone desarrollar hasta el final: la de la evolución de la distribución de la riqueza desde el siglo XVIII hasta nuestros días. En sus primeras líneas Piketty se pregunta si los procesos de acumulación de capital privado nos llevan inevitablemente a una gran concentración de riqueza en unos pocos afortunados, o si por el contrario las fuerzas "invisibles" que acompañan al equilibrio entre crecimiento, competitividad y progreso tecnológico contribuyen tarde o temprano a la reducción de la desigualdad y a una mayor armonía entre las distintas clases sociales. Su proposición recuerda a los grandes debates clásicos a los que Marx, Smith, Keynes y muchos otros dedicaron buena parte de sus escritos, y no por ello deja de ser un proyecto ambicioso. Piketty decidió abordar estas cuestiones desde una perspectiva histórica y comparativa, usando una base de datos extensa ${ }^{3}$ que abarca tres siglos y más de veinte países. Además, utiliza fragmentos de novelas del siglo diecinueve de escritores como Austen o Balzac, como recurso para contextualizar sus datos y explicar cómo funcionaba la sociedad en este período, haciendo referencia a las diferencias de clase y a las desigualdades estructurales que predominaban. Probablemente uno de los aspectos que llama la atención de su discurso, siempre con un toque narrativo que fluye y que recuerda a la narrativa de

\footnotetext{
3 Una de las principales es "The World Top Incomes Database", la cual ha sido la fuente principal de información, al ser la base de datos más extensa hasta el momento en cuanto a la evolución de la desigualdad de rentas.
} 


\section{revista de recerca i formació en antropologia}

\section{perifèria}

Número 19 (2), Diciembre 2014

revistes.uab.cat/periferia

Graeber, es el querer desmarcarse de los métodos econométricos que caracterizan a los economistas convencionales. Tal y como él mismo explica, tras años de estudios matemáticos en EEUU, se dio cuenta de que en realidad, "no sabía nada de los problemas económicos mundiales", y de ahí su decisión de ir a Paris, donde ha acabado dirigiendo el EHEES.

Sin embargo, toda la teoría de Piketty se sustenta sobre una fórmula matemática aparentemente simple, y es que, en el sistema capitalista actual, la tasa de retornos del capital $(r)^{4}$ siempre supera a la tasa de crecimiento de renta $(\mathrm{g})$; es decir, que r>g. Según Piketty ésta es y ha sido siempre la "contradicción central" del capital, y es la lógica irreversible que predomina sobre cualquier otra. Al mismo tiempo, cuando se produce esta tendencia, se produce otro fenómeno; la riqueza heredada crece a una mayor velocidad que la producción y los ingresos. Es decir, "las personas que poseen riqueza heredada sólo necesitan una parte de sus ingresos del capital para ver ese mismo capital crecer a un ritmo más rápido de lo que lo hace la economía en su totalidad" (p.26). Es por esto que la concentración de riqueza se acomoda cada vez más en las clases más altas de la sociedad, donde las rentas a partir del trabajo disminuyen a medida que ascendemos en la escala de ingresos, al mismo tiempo que los ingresos a partir del capital comienzan a interponerse. Hoy, concluye Piketty, uno tiene que ascender más alto dentro de la escala social, para que las rentas del capital sobrepasen a las del trabajo.

No obstante, para comprender bien el significado de esta lógica hay que revisar otros conceptos, probablemente siendo el más importante y uno de los más rebatidos, el del "capital", dado que le atribuye una relación directa con el concepto de "riqueza". Piketty entiende por capital aquellos activos no humanos (pueden ser materiales o inmateriales, como la propiedad intelectual) que pueden ser poseídos e intercambiados en el mercado de forma permanente, ya sean bienes inmuebles o capital financiero y profesional como tierras, infraestructura, maquinaria o patentes (p. 46). Es decir, son "formas de riqueza", que de una manera u otra, pueden ser

4 . " $r$ ", es la ratio anual de retorno de capital (beneficios, dividendos, intereses, rentas y otros ingresos del capital) 


\section{perifèria}

Número 19 (2), Diciembre 2014

revistes.uab.cat/periferia

"cuantificadas" mediante su valor en el mercado (en términos por ejemplo, monetarios, o de acciones). No obstante, Piketty reconoce que en muchas ocasiones no es fácil otorgarle un valor monetario exacto a estos capitales, aunque no le dedica más tiempo a esta cuestión. Es precisamente ésta una de las críticas más fuertes que ha recibido por parte de David Harvey, quien argumenta que el capital no es una "cosa", sino un proceso; "es un proceso de circulación en el cual el dinero se utiliza para crear más dinero a menudo, pero no exclusivamente, a través de la explotación de la fuerza del trabajo" ${ }^{\prime 5}$. Lo que en realidad argumenta Harvey, es que el stock de propiedad privada a la que se refiere Piketty como capital, puede estar siendo utilizado o no. Por tanto, calcular la famosa tasa de retorno del capital ( $r$ ) sobre la cual se basa toda su teoría, es más problemático de lo que parece, ya que todas aquellas fuentes de capital (infraestructuras, fabricas, maquinaria, etc.) que no se usan de forma productiva, están siendo contabilizadas según lo que producen (por tanto hay una relación directa con el índice general de crecimiento), y no según lo que se ha utilizado para su producción.

El economista griego Yanis Varoufakis, también escribe en el Real World Economics Review sobre el concepto de Piketty del capital, y sobre cómo lo ha entrelazado con el concepto de riqueza. Varoufakis entiende el capital como "medios de producción producidos" (p. 19), (produced means of production), y explica que una colección de arte o un garaje lleno de Ferraris, no aportan nada al proceso de producción, puesto que para producir, por ejemplo, un libro fotográfico sobre la colección de arte, se necesitan haber usado personas y tiempo de trabajo en el proceso, por lo que los capitales materiales van necesariamente de la mano del trabajo (2014: 20). Las colecciones de arte y los Ferraris por sí solos, sin embargo, sí cuentan como riqueza, a pesar de no contribuir a la creación de otras mercancías: "el crecimiento de un economía no puede basarse solamente en riqueza, sino que necesita de un tipo de riqueza particular; bienes capitales"...mezclar ambas cosas implica que cuando haya crecimiento de riqueza se dé por hecho que haya habido un

\footnotetext{
${ }^{5}$ Reseña crítica del libro de Piketty, "Afterthoughts on Piketty's Capital" por David Harvey, acceso desde; www.davidharvey.org
} 


\section{perifèria}

Número 19 (2), Diciembre 2014

revistes.uab.cat/periferia

incremento también de la contribución del capital al proceso de producción. Estas dificultades hacen que el concepto del "retorno del capital (r)" sea, una vez más, cuestionado y Varoufakis añade, "si llevamos este argumento algo más lejos, nos podemos preguntar qué lógicas se pueden usar para denominar "retornos de capital" a lo proveniente de bienes físicos o materiales (como puede ser un generador de electricidad) y a denominar "salarios" a los ingresos provenientes de bonos de inversores y gerentes de cartera (p.20).

En cualquier caso, parece haber unanimidad en los conceptos básicos empleados por Piketty. Por un lado, es obvio que el capital ha cambiado. Si hasta el s. XIX se concentraba solamente en las tierras, ahora es industrial, financiero y de bienes inmuebles. Por otro lado, nos encontramos en un periodo marcado por una mitad de la población profundamente pobre, que no posee nada; y con una clase media acaudalada que posee entre un cuarto y un tercio de la riqueza total (p. 377). Asimismo, nos encontramos con el $10 \%$ más rico, que acapara dos tercios del total de la riqueza. La concentración de riqueza sigue siendo alta, aunque no tanto como lo fue hace un siglo. Sin embargo, explica Piketty, la importancia de la riqueza heredada vuelve a tener un protagonismo exacerbado en el siglo XXI, al poder reproducirse más rápidamente que los ahorros generados a partir de las rentas del trabajo, en un clima de poco crecimiento económico.

\section{Crecimiento y shock: Convergencia y Divergencia}

Según Piketty, el capital prosperó durante la década de 1840 y los beneficios industriales crecieron, mientras que las rentas del trabajo se estancaron. Entre 1870 y 1914, la desigualdad se estabilizó en niveles muy altos y siempre estuvo caracterizada por una concentración progresiva de riqueza, la cual no hubiera dejado de aumentar al no ser por el shock económico y político que tiene lugar a raíz de la primera guerra mundial. Fueron precisamente el auge de la producción masiva industrial y la precariedad de los trabajadores en las fábricas lo que alentó a Marx y a Engels a escribir The Conditions of the Working Class in England (1844) y lo que propició, a posteriori, el auge del comunismo y del socialismo. De hecho, 


\section{perifèria}

Número 19 (2), Diciembre 2014

revistes.uab.cat/periferia

Piketty no ignora el peso de la política en la regulación de la economía y la distribución de la riqueza. Por el contrario, argumenta que precisamente fueron las políticas regulatorias que se llevaron a cabo entre 1910 y 1950 -y que fueron la consecuencia, no solo de las guerras, sino también de las políticas que se adoptaron para paliar los efectos devastadores- lo que redujo la desigualdad durante dicho período. Asimismo, tiene en cuenta la difusión del conocimiento y la mejora de la educación como el factor que más ha contribuido a la reducción de la desigualdad a la larga. No obstante, en muchos casos esta fuerza de convergencia no es suficiente para paliar la tendencia a la divergencia; la cual se puede dar perfectamente (como hemos podido observar recientemente) en condiciones de mercado eficientes, y puede resultar más poderosa que las fuerzas convergentes. Esto es precisamente lo que vuelve a ocurrir a partir de 1979 con el gobierno de Margaret Thatcher y Ronald Reagan, cuando el capital vuelve a crecer significativamente como lo había hecho en 1913.

Piketty ejemplifica su teoría con el caso de Estados Unidos, cuya serie histórica de datos abarca desde 1915 a 2012. En la gráfica se puede observar cómo a partir de la década de los 80 la desigualdad comienza a crecer exponencialmente y cómo durante la Edad de Oro del capitalismo (1945-1973) se mantiene más estable y a niveles inferiores. Esto se debe a que durante este período se pusieron en marcha políticas de distribución que favorecían el mercado de trabajo y que hicieron que los ingresos a nivel individual se volvieran más equitativos. En realidad fueron políticas que desfavorecían la acumulación de capital en un periodo de la historia en el que debido a las guerras, se produjo una destrucción física y masiva de bienes capitales, al mismo tiempo que se aplicaban impuestos de patrimonio e impuestos confiscatorios a los ingresos más altos para paliar los efectos. En definitiva, tras la II Guerra Mundial, el apoyo al trabajador fue más pronunciado y esto actuó en detrimento del capital. En términos de Piketty, la ratio capital/ingresos ( $\beta$ ) se vio disminuido, por lo que hay una menor concentración de capital y más equidad de ingresos personales. Por el contrario, si tenemos en cuenta períodos como los de Margaret Thatcher en Reino Unido, donde la desigualdad comienza también a ascender en la década de los 70, nos encontramos con que el capital se había 


\section{perifèria}

Número 19 (2), Diciembre 2014

revistes.uab.cat/periferia

regenerado considerablemente, provocando la subida de $\beta$ y crecimiento de las fuerzas de divergencia. La tendencia había cambiado y el respaldo estaba en el capital, no en el trabajo.

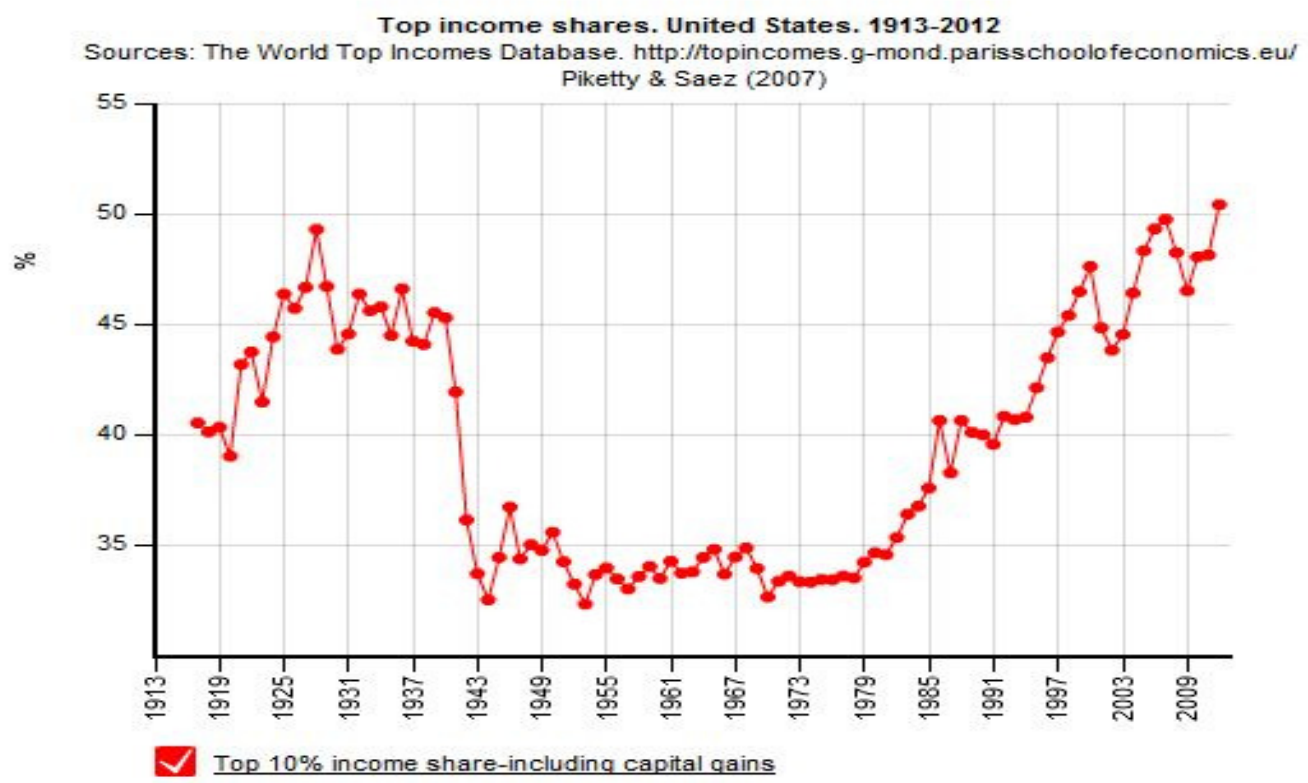

Tabla 1. Desigualdad de Ingresos en Estados Unidos, 1913-2012

\section{Desigualdad de sueldos: Educación y tecnología}

Tras presentar el marco macro de la desigualdad, Piketty se embarca en la tarea de explicar en detalle la estructura de la desigualdad; es decir, a encontrar los mecanismos sistemáticos que dan lugar a dichas disparidades a nivel nacional. Con este fin, Piketty comienza el capítulo noveno explicando que la desigualdad de sueldos en diferentes países y periodos de tiempo no puede explicarse solamente en términos de productividad marginal, sino que las instituciones y las normas que regulan el mercado laboral tienen un peso significativo en los resultados (p.308). La evolución de los salarios mínimos es social, política y cultural; depende de cada país. Sin embargo, a pesar de la gran inversión que ha habido, por ejemplo, en 


\section{perifèria}

Número 19 (2), Diciembre 2014

revistes.uab.cat/periferia

EEUU con respecto al acceso a la educación superior y a las nuevas tecnologías, la desigualdad de ingresos particulares sigue disparándose en la década de los 80 . Uno de los ejemplos que propone Piketty para explicar a qué se deben estas diferencias, es la del auge de la cultura del "supermanager" (p. 315) en el ámbito anglosajón, tanto en el sector financiero como fuera de él. La tolerancia a los sueldos desorbitados de los ejecutivos se exacerbó durante la década de los 70 y los 80. Probablemente una de las mejores contribuciones etnográficas que se han hecho en este respecto es la de Karen Ho (2009), "Liquidated: An Ethnography of Wall Street", donde relata precisamente cómo se establecen las escalas de valores a través de las cuales se eligen sueldos y compensaciones en general dentro de las grandes firmas y corporaciones. Como también resalta Piketty, y explica Ho, el acceso más libre a la educación superior ha coincido con el auge de centros elitistas de los cuales salen estos inversores. Estos espacios de trabajo reflejan perfectamente esta jerarquía de valores y de diferenciación salarial y de estatus ( $p$. 11). De esta manera, se ha propiciado la subida de ingresos totales del porcentaje más rico de la población de forma mucho más pronunciada que en el resto de países de Europa continental y Japón. Por tanto, Piketty atribuye la desigualdad de ingresos particulares en parte a las leyes econométricas de la productividad marginal y a las inversiones en educación y tecnología (en cuanto a la evolución de los sueldos a largo plazo), pero también al nacimiento de una cultura permisible con las jerarquías dentro de empresas y corporaciones.

\section{Conclusión}

Posiblemente la parte del libro de Piketty que más ha sido criticada, o descrita como utópica, ingenua y descabellada, ha sido su propuesta de implantar un sistema de tributación progresiva- junto con impuestos sobre sucesiones, según los ingresos particulares- como solución a la creciente desigualdad. Es decir, su solución no es de ninguna manera revolucionaria en el sentido marxista, sino más bien "regulatoria". Piketty no está en contra del sistema capitalista, como ya ha aclarado en varias entrevistas tras la publicación del libro, sino que está a favor de 


\section{perifèria}

Número 19 (2), Diciembre 2014

revistes.uab.cat/periferia

ponerle límites a la "mano invisible" que (presuntamente) equilibra los mercados.

A pesar de las numerosas críticas que ha recibido, el valor de la contribución de Piketty es innegable. Por un lado, intentar reconciliar la perspectiva económicaneoclásica mediante las leyes econométricas con los aspectos sociales y políticos, a pesar de haber profundizado poco en estos. Y por otro lado, el haber utilizado y creado nuevas formas de analizar datos extensos que nos hablen sobre la riqueza y la desigualdad. Probablemente lo más interesante del trabajo de Piketty, y lo que más haya podido resonar a los ecos de Marx, haya sido su interés por identificar las contradicciones internas del capitalismo y por analizar patrones o tendencias teniendo en cuenta el contexto y la evolución histórica del sistema para, como aduce también Ghosh (2014), "explicar tendencias capitalistas contemporáneas" ( $p$. 139). El mensaje de Piketty es, además, claro: advierte de que si tenemos en cuenta las mayores riquezas del mundo, alrededor del $60-70 \%$ de estas se componen de patrimonio heredado y la tendencia de este porcentaje es a seguir aumentando (p. 443), por lo que podríamos fácilmente volver a las cifras de la Belle Époque (1870-1914), durante la cual las herencias componían el 80-90\% de la riqueza total acumulada de los individuos.

La cultura emprendedora de nuestro tiempo (pensemos en Sillicon Valley y en las fortunas de emprendedores como Marc Zuckerberg, Steve Jobs, o herederos y herederas de grandes multinacionales, cuyas fortunas se siguen incrementando año tras año), junto con la tendencia $r>g$, un crecimiento económico bajo y una desigualdad de retornos del capital, nos lleva inevitablemente a un incremento automático de capital inicial, que provocará que las fortunas crezcan y se perpetúen hasta límites que sobrepasen la utilidad social. De no ser así, Piketty avisa de que solo catástrofes como las que hemos vivido en el $s$. XX podrían frenar esta tendencia.

Aunque la perspectiva de Piketty siga siendo meramente económica, en el sentido de que toda su teoría se basa en una ley econométrica neoliberal "fundamental" que explica los desequilibrios que desencadenan la concentración de riqueza, no deja de ser de gran valía como marco histórico-conceptual. Aunque el tema de la desigualdad social ha sido y sigue siendo un tema recurrente para sociólogos y 


\section{perifèria}

Número 19 (2), Diciembre 2014

revistes.uab.cat/periferia

antropólogos, la obra de Piketty logra situar los mecanismos macro-estructurales en términos de poder bajo una lógica empírica y global que puede ser de mucha utilidad para comprender las lógicas que influyen en las acciones individuales y colectivas de la sociedad. Al igual que hizo David Graeber en La Deuda: Los primeros 5000 años (2011), Piketty logra analizar una gran cantidad de datos empíricos e históricos y explicarlos bajo una lógica mucho más holística y comparativa de lo que los economistas venían haciendo hasta ahora.

Desde la perspectiva de la antropología de la economía, Stephen Gudeman insiste en la necesidad de recordar que el mercado, como ya había mencionado anteriormente Durkheim, es un ente socialmente constituido y que por tanto, hay normas y valores culturales tras los mecanismos que rigen su funcionamiento (p.16). Sin embargo, añade Gudeman, "Piketty relega el cómo se establecen estas normas y valores al plano de la psicología, la sociología, etc...en vez de tratarlos como parte de la economía [...] la cual se compone de relaciones sociales, intercambios impersonales, e instituciones" (2014: 16). Es por esto que a la pregunta del por qué son los retornos del capital mayores que la ratio de crecimiento, la respuesta de Piketty alegando que simplemente los datos y la historia así lo avalan, sin ser necesariamente algo lógico (p. 353), no es suficiente para un antropólogo.

Tal y como ya venían explicando otros académicos como Zygmunt Bauman (Trabajo, Consumo y Nuevos Pobres, 1999; Daños Colaterales: Desigualdades sociales en la Era Global, 2011), Boltanski y Chiapello (El Nuevo Espíritu del Capitalismo, 2002), Senet (La Cultura del Nuevo Capitalismo, 2006) o Standing (El Precariado, 2011), entre muchos otros, el desarrollo del mercado laboral, el papel de los Estados e instituciones como "benefactores" a la vez que opresores, y la condición actual de los individuos como meros "consumidores", conlleva a replantearse cuestiones como la identidad, la salud, la cultura de socialización, o la nueva pobreza. Como Wilkinson y Pickett (2009) explican, la desigualdad no se queda en disparidad de rentas, sino que afecta a la confianza (p. 72), se incrementa la importancia del estatus (p. 157), y aumentan los problemas sociales en general. Todo esto es parte del constructo social que son los mercados, y por 


\section{revista de recerca i formació en antropologia}

\section{perifèria}

Número 19 (2), Diciembre 2014

revistes.uab.cat/periferia

tanto, es imposible explicar una cosa sin la otra, de la misma manera que es imposible hablar de lo "global" sin hacer referencia a lo "local", y viceversa. Las estructuras de poder que se legitiman y que forman el engranaje de la sociedad no conforman lógicas "naturales", sino construidas, y en este aspecto, la antropología todavía tiene mucho que decir.

\section{Bibliografía}

Bauman, Z. (1999). Trabajo, Consumo y Nuevos Pobres. Barcelona: Gedisa

Bauman, Z. (2011). Daños Colaterales: Desigualdades Sociales en la Era Global. Madrid: FCE

Boltanski, L. y Chiapello, E. (2002). El Nuevo Espíritu del Capitalismo. Madrid: Akal Ghosh, J. (2014). 'Piketty and the Resurgence of Patrimonial Capitalism', RealWorld Economics Review, 69

Gudeman, S. (2014). 'Piketty and Anthropology', Anthropological Forum: A Journal of Social Anthropology and Comparative Sociology

Gregory, C. (2014). 'The Three Faces of Thomas Piketty: Reflections on a Number 1 best-seller', Anthropology of This Century, 11 (acceso online http://aotcpress.com/articles/faces-thomas-piketty-reflections-1-bestseller/, 01-112014)

Harvey, D. (2014). 'Afterthoughts on Piketty's Capital', http://davidharvey.org/2014/05/afterthoughts-pikettys-capital/ (acceso online 0111-2014)

Ho, Karen. (2009). Liquidated: An Ethnography of Wall Street. Durham, London: Duke University Press

Piketty, T. (2014). Capital in the 21st Century. London: The Belknap Press of Harvard University Press

Sennett, R. (2006). La Cultura del Nuevo Capitalismo. Barcelona: Editorial 


\section{perifèria}

Número 19 (2), Diciembre 2014

revistes.uab.cat/periferia

Anagrama, S. A.

Standing, G. (2011). El Precariado: Una Nueva Clase Social. Barcelona: Ediciones de Pasado y Presente, S.L.

Varoufakis, Y. (2014). 'Egalitarianism's Latest Foe', Real-World Economics Review, 69

Wade, R. (2014). 'The Piketty Phenomenon and the Future of Inequality', RealWorld Economics Review, 69

Wilkinson, R. y Pikckett, K. (2009). Desigualdad: Un Análisis de la (in)felicidad colectiva. Madrid: Turner Publicaciones

The World Top Incomes Database: http://topincomes.parisschoolofeconomics.eu/ 